\title{
Localisation of factor XIII in human tissues using an immunoperoxidase technique
}

\author{
JD FEAR,${ }^{*}$ P JACKSON,$\dagger$ C GRAY,$\dagger$ KJA MILOSZEWSKI, ${ }^{*}$ MS LOSOWSKY* \\ From the *Department of Medicine, St James's University Hospital, Leeds LS9 7TF, and the †Department of \\ Pathology, University of Leeds, Leeds LS2 9JT
}

SUMMARY An immunoperoxidase technique has been used to localise clotting factor XIII subunits $A$ and $S$ in human tissues. The presence of factor XIII in placenta and megakaryocytes was confirmed. Factor XIII was also found in fibroblasts, a hitherto unreported finding. Factor XIII subunits were not detected in hepatocytes, although factor XIII was found in fibroblasts in portal tracts. These findings suggest that factor XIII is not synthesised in the liver as previously thought.

Factor XIII is a plasma proenzyme which circulates as a dimer of the active subunit (subunit $\mathrm{A}$ ) attached to a dimer of the carrier protein (subunit S). It is essential for normal clot formation and inherited factor XIII deficiency results in a serious bleeding disorder. Factor XIII concentrations are low in conditions in which hepatic protein synthesis is impaired, such as chronic liver disease, ${ }^{1}$ and raised in conditions in which hepatic protein synthesis is enhanced, such as the nephrotic syndrome ${ }^{2}$ and the hypertriglyceridaemias. ${ }^{3}$ Consequently, factor XIII is thought to be synthesised in the liver. It has been found in megakaryocytes in bone marrow and in cells in the placenta by immunofluorescence ${ }^{4}$ and Ikematsu $^{5}$ has reported that factor XIII can be detected in hepatocytes using a immunoperoxidase technique. There is, however, little correlation between tests of liver function and plasma factor XIII concentrations, and concentrations can also be low in conditions which do not affect the liver. ${ }^{6}$

Since the site of synthesis of factor XIII is in doubt we have studied the localisation of subunits $A$ and $S$ in human tissues, including liver, bone marrow, and placenta, using an immunoperoxidase technique with subunit specific antisera.

\section{Material and methods}

Rabbit antihuman antiserum to subunits $A$ and $S$ was obtained from Hoechst (Clotimmun, Behringwerke, batch nos 105813A, 105904A). Peroxidase conjugated swine antirabbit antiserum was obtained from Dako (Mercia). All other material was obtained from BDH.

\section{TISSUES}

Paraffin embedded human tissue was obtained from the surgical pathology files of a department of histopathology. Tissues examined included four histologically normal specimens of liver obtained by surgical and needle biopsy or at necropsy, hypo- and hypercellular bone marrow trephine biopsy specimens, normal full term placenta, and a surgically excised partial gastrectomy specimen containing a benign peptic ulcer. In addition, fresh liver and placenta were obtained for both paraffin embedding and frozen sections.

\section{METHOD}

Tissue blocks were fixed in phosphate buffered formalin for at least $24 \mathrm{~h}$ and then processed by standard methods.

1 Sections $(4 \mu \mathrm{m})$ were taken to water via xylene and alcohol.

2 Endogenous peroxidase activity was blocked by immersing the sections in $1 \%$ hydrogen peroxide in absolute methanol for $30 \mathrm{~min}$.

3 Sections were treated with $0.1 \%$ trypsin in $0.1 \%$ calcium chloride in TRIS buffered saline (TBS) at $37^{\circ} \mathrm{C}, \mathrm{pH} 7 \cdot 6$, for $20 \mathrm{~min}$ with a subsequent wash in TBS (5 min).

4 Sections were then treated with a 1/5 dilution of normal swine serum (NSS) in TBS at room temperature in a humid chamber for $10 \mathrm{~min}$.

5 Excess NSS/TBS was removed, and 1/25 rabbit antihuman factor XIII subunit A or S antiserum (in $1 / 20 \mathrm{NSS} / \mathrm{TBS}$ ) was added and the sections incubated in a humid chamber at room temperature for $15 \mathrm{~min}$.

6 Sections were washed in TBS for $5 \mathrm{~min}$ and incubated with $1 / 25$ swine antirabbit IgG peroxidase 
conjugate (in 1/20 NSS/TBS, $\mathrm{pH} \mathrm{7.6)} \mathrm{at} \mathrm{room} \mathrm{temp-}$ erature for $15 \mathrm{~min}$, with a subsequent wash in TBS for $10 \mathrm{~min}$.

7 The sections were then rinsed in $0.05 \mathrm{M}$ acetate buffer $\mathrm{pH} 5$ for $30 \mathrm{~s}$ and incubated (stirring) in $0.02 \%$ amino-ethyl carbazole in $0.05 \mathrm{M}$ acetate buffer $\mathrm{pH} 5$ for up to $1 \mathrm{~min}$.

8 Hydrogen peroxide was added to a final concentration of $0.02 \%$ and the sections were incubated for a further 5-10 min.

9 Finally, the sections were rinsed with $0.05 \mathrm{M}$ acetate buffer $\mathrm{pH} 5$ and tap water, counterstained with Harris's haematoxylin, and mounted in neutral glycerine jelly.

\section{CONTROLS}

Negative control. Omission of primary antiserum (anti-A or anti-S) abolished staining.

Adsorption control. Anti-A antiserum was adsorbed with factor XIII concentrate (Fibrogammin, Hoechst, contains only subunit A) by mixing the antisera with the concentrate; absorption abolished staining.

Extinction of staining with progressive dilution of the primary antisera. Staining was progressively reduced until extinction occurred at titres of $1 / 200$ for anti-A and anti-S.

Omission of trypsinisation did not abolish staining for both subunit $A$ and subunit $S$ but did result in a diminution of staining intensity.

\section{Results}

Intense positive staining for subunits $A$ and $S$ was found in spindle and stellate cells situated in the fibrous connective tissue of the portal tracts. There was no staining with either anti-A or anti-S antiserum in hepatocytes. In addition, staining was absent in Küpffer cells, capillary and sinusoidal endothelium, bile duct epithelium, lymphoid cells, and erythrocytes. The positively stained cells are interpreted as inactive fibroblasts (Fig. 1).

In both hypo- and hypercellular marrow there was intense staining of megakaryocytes with both antisera; all other cells, including osteoblasts and capillary endothelium, were not stained.

In placenta, intense positive staining for subunits $A$ and $S$ was found exclusively in the mesenchymal cells of the connective tissue of the chorionic villi. Syncytiotrophoblast, cytotrophoblast, and vascular

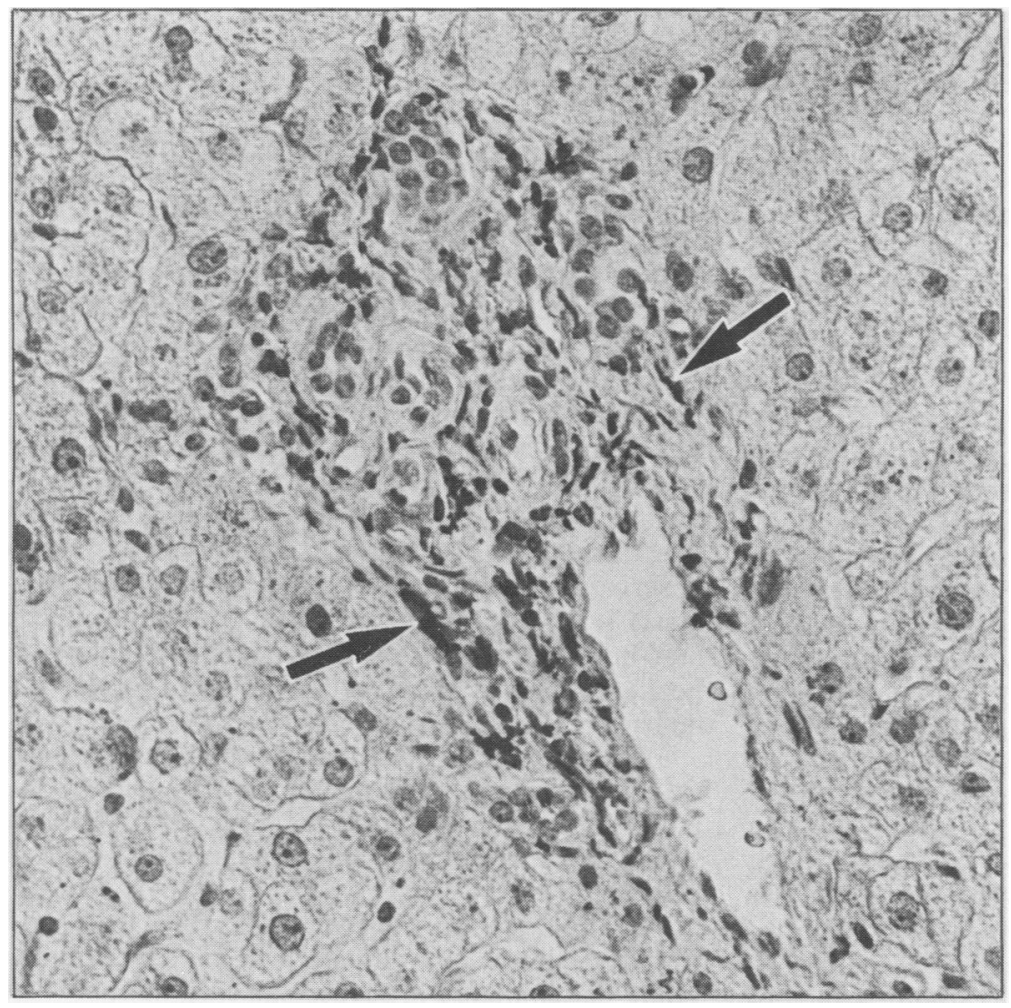

Fig. 1 Positive staining (arrows) for factor VIII (subunit A) in fibroblasts in the portal tract of a wedge biopsy specimen of human liver. Neither subunit $A$ nor subunit $S$ were shown in hepatocytes. This staining pattern was consistently reproducible in all liver specimens (see text). The liver was histologically normal. $\times 750$. 


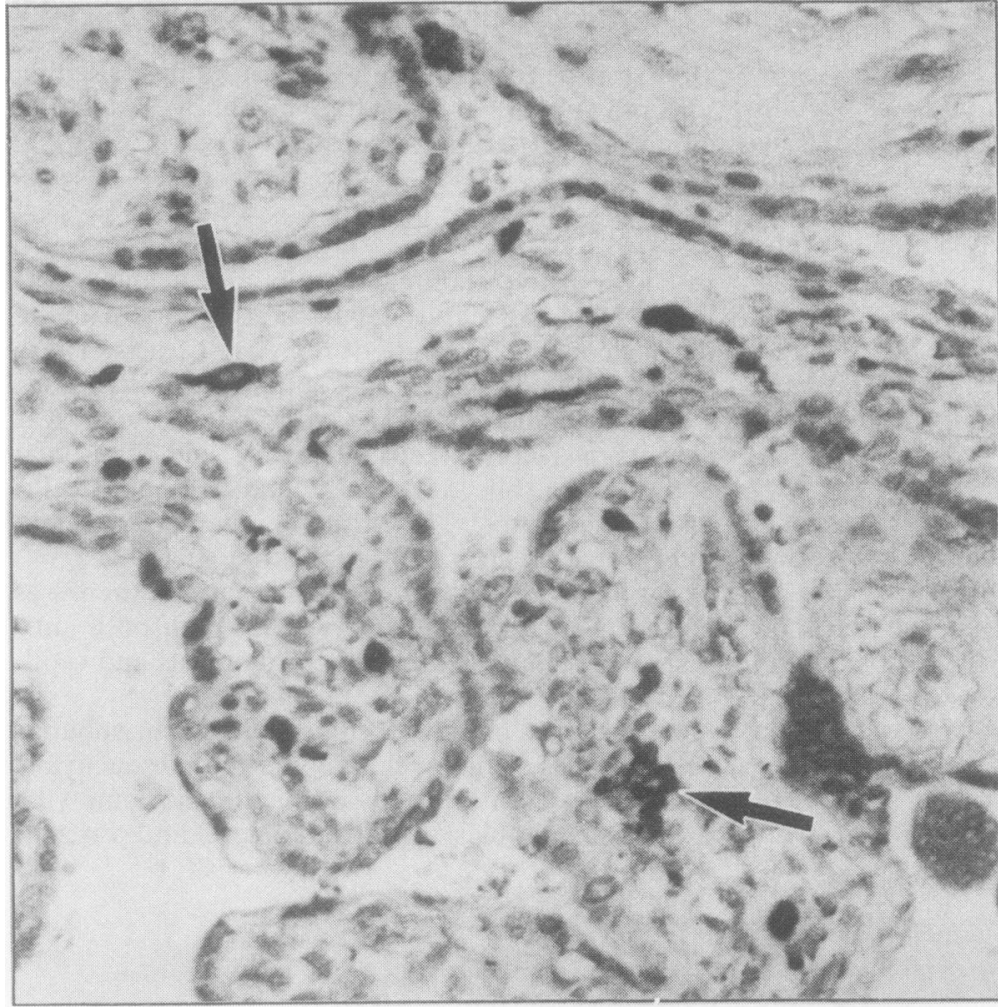

Fig. 2 Positive staining for factor $X I I I$ (subunit A) in mesenchymal cells (arrows) in a chorionic villus of a full term placenta. Positive staining of both subunit $A$ and subunit $S$ was found. Syncitiotrophoblast and cytotrophoblast were not stained. $\times 750$.

endothelium were all negative (Fig. 2).

The partial gastrectomy specimen showed positive staining for subunits $A$ and $S$ in active fibroblasts in the zone of granulation tissue subjacent to the chronic ulcer. Staining was absent in gastric mucosal epithelium, smooth muscle, and vessels.

\section{Discussion}

Using an immunoperoxidase technique employing subunit specific factor XIII antisera we have been able to show specific staining of factor XIII subunits $A$ and $S$ in human tissues. Subunit $A$ was found in megakaryocytes and placenta as has been previously described, ${ }^{4}$ and we were also able to show subunit $\mathrm{S}$ in these tissues, a finding not previously noted. Bohn et $a l^{7}$ have shown that factor XIII extracted from human placenta has a similar molecular weight to subunit A; plasma factor XIII has a much larger molecular weight owing to the combination of subunits $A$ and $S$. Subunits $A$ and $S$ are both present in the cells but may not be combined as in the plasma, which may explain why subunit $\mathrm{S}$ has not previously been found in placenta. The staining of the two subunits in our material was cytoplasmic in distribution.

We were unable to show either subunit $A$ or sub- unit $S$ in the hepatocytes of human liver obtained in three different ways, even when using antisera at titres which gave reproducible specific staining in other cells in the same section. Ikematsu ${ }^{5}$ has reported that the two subunits can be detected in hepatocytes, but the validity of this report is questionable as details of the method used, controls, antisera titres, and source of the tissue are not given. Lee and Chung ${ }^{8}$ have reported the detection of factor XIII in isolated rabbit hepatocytes, but again insufficient details were given for adequate assessment of the report. The positive staining of connective tissue cells interpreted as fibroblasts is a new observation. Similar cytoplasmic staining of fibro- No blasts was seen at the site of a peptic ulcer. This $N$ may be relevant to the role of factor XIII in wound healing, which is abnormal in inherited factor XIII deficiency: ${ }^{\circ}$

Of course, factor XIII may be present in hepatocytes in amounts too small to be detected by this immunoperoxidase technique, but the amounts would be appreciably smaller than in the fibroblastic cells.

The low plasma concentrations of factor XIII found in chronic liver disease may be due to the low grade disseminated intravascular coagulation which 

occurs in these patients. ${ }^{9}$ Plasma factor XIII concentrations are known to fall in disseminated intravascular coagulation. ${ }^{10}$ Increased concentrations of factor XIII in the nephrotic syndrome may be due to increased marrow turnover since these patients can have thrombocytosis," and this could also account for raised factor XIII concentrations in hyperlipidaemias. Some patients with hyperlipidaemias have increased platelet turnover and hence increased megakaryocytic activity. ${ }^{12}$

This study shows that factor XIII is not present in human hepatocytes and suggests that plasma factor XIII may be produced by megakaryocytes or fibroblasts. The demonstration of factor XIII in fibroblasts is new and may provide a link with previous observations of poor wound healing in patients with inherited factor XIII deficiency. It would be of interest to see if factor XIII could be detected in human fibroblasts in vitro.

We thank Professor CC Bird for his assistance.

\section{References}

' Biland L, Duckert F, Prisender S, Nyman D. Quantitative estimation of coagulation factors in liver disease. The diagnostic and prognostic value of factor XIII, factor $\mathrm{V}$ and plasminogen. Thromb Haemostas 1979;39:646-56.

${ }^{2}$ Yoshioka K, Miyata H, Uraoka Y, Maki S. Plasma factor XIII levels in children with renal disease. Nephron 1981;27:19-24.
${ }^{3}$ Cucuianu MP, Miloszewski K, Porutiu D, Losowsky MS. Plasma factor XIII and platlet factor XIII in hyperlipaemia. Thromb Haemostas 1976;36:542-50.

4 Kiesselbach TH, Wagner RH. Demonstration of factor XIII in human megakariocytes by fluorescent antibody technique. Proc N Y Acad Sci 1972;202:313-28.

${ }^{5}$ Ikematsu S. An approach to the metabolism of factor XIII. Acta Haematol Jpn 1981;44:1499-505.

- Miloszewski KJA, Losowsky MS. Clinical consequences of inherited and acquired factor XIII deficiency. In: Egbring $\mathbf{R}$, Klingemann HG, eds. Factor XIII and fbronectin. Marburg/ Lahn: Die Medizinische Verlagsgesellschaft $\mathrm{mbH}, 1983: 31-$ 40.

' Bohn H, Haupt H, Krauz T. Die molekulare struktur der fibrinstabilisierenden faktoren des menschen. Blut 1972;25:235-48.

${ }^{8}$ Lee SY, Chung SI. Biosynthesis and degradation of plasma protransglutaminase (factor XIII). Fed Proc 1976;35:1486.

9 Bloom AL. Intravascular coagulation and the liver. $\mathrm{Br} J$ Haematol 1975;30:1-6.

${ }^{10}$ Rodeghiero F, Barbui T, Battista R, Chisesi T, Rigoni G, Dini E. Molecular subunits and transamidase activity of factor XIII during disseminated intravascular coagulation in acute leukaemia. Thromb Haemostas 1980;43:6-9.

" Adler AJ, Lundin AP, Fienroth MV, Friedman EA, Berlyne GM. $\beta$ Thromboglobulin levels in nephrotic syndrome. Am J Med 1980;69:551-4.

12 Carvalho ACA, Colman RW, Lees RS. Platelet function in hyperlipoproteinemia. $N$ Engl J Med 1974;290:434-8.

Requests for reprints to: Dr JD Fear, Department of Medicine, St James's University Hospital, Leeds LS9 7TF, England. 